\title{
ILCEA
}

Revue de l'Institut des langues et cultures

d'Europe, Amérique, Afrique, Asie et Australie

29 | 2017

Les femmes en Russie : parcours, mythes et représentations

\section{Aux côtés des génies - Dostoïevskaïa et Tolstaïa}

ЖИЗНЬ РЯДОМ С ГЕНИЕМ - АННА ДОСТОЕВСКАЯ И СОФЬЯ ТОЛСТАЯ

\section{Christa Ebert}

\section{(2) OpenEdition}

Journals

Édition électronique

URL : http://journals.openedition.org/ilcea/4286

DOI : 10.4000/ilcea.4286

ISSN : 2101-0609

\section{Éditeur}

UGA Éditions/Université Grenoble Alpes

\section{Édition imprimée}

ISBN : 978-2-37747-007-5

ISSN : 1639-6073

\section{Référence électronique}

Christa Ebert, «Aux côtés des génies - Dostoïevskaïa et Tolstaïa », ILCEA [En ligne], 29 | 2017, mis en ligne le 30 juin 2017, consulté le 23 avril 2019. URL : http://journals.openedition.org/ilcea/4286 ; DOI : 10.4000/ilcea.4286

Ce document a été généré automatiquement le 23 avril 2019

(C) ILCEA 


\title{
Aux côtés des génies - Dostoïevskaiia et Tolstaïa
}

\author{
ЖИЗНЬ РЯДОМ С ГЕНИЕМ - АННА ДОСТОЕВСКАЯ И СОФЬЯ ТОЛСТАЯ
}

\section{Christa Ebert}

1 À propos du livre Deux épouses. Tolstaïa et Dostö̈evskaïa [Dve ženy. Tolstaâ i Dostoevskâ.. Materialy $i$ kommentarii] publié par Yuri Aichenwald en 1925 à Berlin, Zinaida Guippius pose la question suivante :

КАКОЙ ДОЛЖНА БЫТЬ В ИДЕАЛЕ, ЖЕНА ЗАМЕЧАТЕЛЬНОГО ЧЕЛОВЕКА, ГЕНИАЛЬНОГО ПИСАТЕЛЯ? БЕЗЗАВЕТНО ПРЕДАННОЕ ЖЕНСКОЕ СУЩЕСТВО, НЯНЬКА, ЛЮБЯЩАЯ КУХАРКА, СЛОВОМ - САМООТВЕРЖЕННАЯ «СЛУЖИТЕЛЬНИЦА ГЕНИЯ? (1991 : 215)

Elle répond à cette question dans l'article «O ženah» [« À propos des épouses »]: la femme idéale pour un artiste n'existe pas, mais il existe des couples heureux et d'autres malheureux.

2 Comme exemple de mariage malheureux, elle cite celui de Pouchkine et Gontcharova, et comme couple heureux celui de Tourguenïev et Pauline Viardot, malgré le fait, ou grâce au fait qu'ils n'étaient pas mariés. Guippius mesure le bonheur de l'écrivain dans ses deux hypostases : l'homme et l'artiste. Dans son article, elle compare la vie d'Anna Grigorievna Dostoïevskaïa avec celle de Sofia Andreïevna Tolstaïa, et elle exprime son respect pour l'une comme pour l'autre, pour leur façon de mener leur vie aux côtés de deux maris assez compliqués. Elle s'intéresse à la situation de la femme seulement en référence au bien-être de l'homme, de l'homme créatif, bien sûr. Mais la femme elle-même, n'a-t-elle pas le droit d'être considérée comme une individualité propre ? Il est intéressant de voir que cette question est posée non par Guippius, mais par Yuri Aichenwald, qui fait dans son livre un plaidoyer en faveur des femmes des grands écrivains contre leurs diffamateurs. Il se dresse tout particulièrement contre le célèbre ouvrage de Vladimir Chertkov: Uhod Tolstogo [La fuite de Tolstoï], où celui-ci reproche à Sofia Andreïevna d'avoir entravé la liberté du génie en mettant en avant sa personnalité autonome :

[...] ИНТЕРЕСНА БЫЛА ЭТА ОДАРЕННАЯ ЖЕНЩИНА САМА ПО СЕБЕ, А НЕ ТОЛЬКО В ОТРАЖЕННОМ СВЕТЕ СВОЕГО СУПРУГА. (Ajhenval'd, 1925 : 134)

Quant à Dostoïeskaïa, il la voit plutôt comme une sainte martyre : 
СПОКОЙНАЯ, ЯСНАЯ, С ЧИСТЫМ СЕРДЦЕМ, РАЗДЕЛЯЕТ ОНА ЕГО ЖИЗНЬ И ВСЕ ЕГО БЕСЧИСЛЕННЫЕ ТЕРЗАНИЯ. (1925 : 140)

Et de plus :

[...] ВЧУЖЕ СТАНОВИТСЯ ЖУТКО ОТ ЭТОГО ЗРЕЛИЩА ТАКОЙ СОВМЕСТНОСТИ, В КОТОРОЙ ДЛЯ ЖЕНЫ НИ ОДНА МИНУТА НЕ БЫЛА ОБЕСПЕЧЕНА ОТ ЯРОСТНЫХ ВСПЫШЕК ПУСТЬ ГЕНИАЛЬНОГО, НО И МАНИАКАЛЬНОГО МУЖА. (1925 : 146)

4 Si on compare son jugement sur Tolstaïa avec celui qu'on trouve dans une biographie récente datant de 2010 , on peut conclure à un retour actuel au modèle traditionnel (Nikitina, 2010). Cette nouvelle évocation de la vie de Tolstaïa est riche en détails biographiques, mais l'héroïne est traitée exclusivement dans sa fonction d'épouse du grand poète et non comme personnalité autonome. Dans ce livre, son attitude semble vue uniquement en fonction des besoins de son célèbre mari : Sofia est valorisée quand elle accompagne les revendications de son mari, et mauvaise quand elle s'oppose à lui.

5 On est en droit d'estimer, dans un premier temps, qu'il est injuste et réducteur de se contenter de cette image de Sofia Andreevna comme épouse de l'écrivain célèbre. Toutefois, il faut bien reconnaître qu'elle-même a très vite accepté et endossé ce rôle de personnage public, celui d'épouse du grand Tolstoï.

6 Toute sa correspondance, ses journaux et ses textes autobiographiques témoignent de sa conscience de n'être plus qu'une personnalité publique. Il n'y a plus de vie individuelle et privée pour Sofia Andreïevna après son mariage.

7 Il en est de même pour Anna Grigorievna Dostoïevskaïa, mais la façon dont se déroule la transformation de la jeune fille en épouse d'un homme de génie est complètement différente. Les deux femmes ont en commun un statut et un destin qui les a amenées à vivre aux côtés d'hommes de génie, mais en même temps elles ont des idées différentes sur leur rôle personnel dans cette situation moitié publique - moitié privée.

Zinaida Guippius les a rencontrées toutes les deux, Dostoïevskaïa et Tolstaïa, et elle s'intéresse surtout à la relation avec leurs maris. Dans ce contexte elle constate que Dostoïevskaïa se considérait comme épouse et, plus tard, comme veuve, d'un grand écrivain, plutôt que comme maîtresse de maison ou mère de ses enfants. En premier lieu elle aimait l'œuvre, c'est-à-dire qu'elle préférait l'écrivain à l'homme. Et il en était de même pour lui. Il aimait sa femme, mais ce n'était pas une relation très passionnée ; elle était d'abord son assistante, et au-delà, avec son esprit pratique, elle lui offrait les conditions d'une vie familiale relativement normale. Ce dernier point avait une grande importance, en raison de son caractère difficile. Guippius constate :

АННА ГРИГОРЬЕВНА - ИЗ ТЕХ «ЛУЧШИХ» ЖЕН, «СЛУЖИТЕЛЬНИЦ ГЕНИЯ», В БРАКЕ С

КОТОРЫМИ ВЕЛИКИЕ НАХОДЯТ ВОЗМОЖНОЕ ДЛЯ НИХ «СЧАСТЬЕ». (1991 : 217)

Et elle ? Était-elle heureuse ? Guippius ne pose pas cette question, et elle ne se demande ni qui est cette femme, ni ce qu'elle représente. La femme d'un poète ou d'un écrivain occupe naturellement une position intermédiaire et ambiguë : elle a une vie publique dans sa fonction de femme de poète - et en tant que telle elle est considérée et jugée par les historiens de la littérature. En même temps, sa place est dans la vie quotidienne, où elle doit organiser la vie de famille, le confort et les conditions agréables pour son mari, et dans cette fonction elle partage le destin de toutes les femmes de son milieu et de son époque.

Il ne s'agit pas ici de discuter le jugement porté par la critique ou par la presse sur le comportement des femmes des poètes, mais de regarder les conditions familiales dans 
lesquelles les deux femmes exprimaient leur personnalité et comment elles cherchaient et trouvaient leur place dans la vie quotidienne.

\section{L'épouse de Dostoïevski}

11 Anna Grigorievna Snitkina (1846-1918) était la fille d'un fonctionnaire d'État d'origine ukrainienne et d'une Suédoise de deuxième génération qui vivait à Saint-Pétersbourg. Elle reçut une éducation remarquable pour une femme de son temps : après l'école allemande Sainte-Anne, elle entra au premier lycée de jeunes filles, qui ouvrit ses portes à SaintPétersbourg en 1858. Elle termina ses études avec la médaille d'argent. Ensuite elle fut admise aux cours pédagogiques de Vyshnegradsky où elle suivait les enseignements de physique et de mathématiques. À cette époque, elle s'intéressait aussi beaucoup à la littérature et lisait des romans contemporains. Elle se considérait comme une femme moderne des années soixante ${ }^{1}$. Quand son père tomba malade, elle dut quitter l'institut pour le soigner, mais trouva une possibilité de continuer sa formation: lorsqu'elle parvenait à se libérer de la charge de son père, elle suivait les cours de sténographie de P. M. Olchine qui avaient lieu le soir. Le professeur Olchine appréciait tant son élève Snitkina qu'il la recommanda à Dostoïevski, qui cherchait une sténotypiste pour écrire son roman Lejoueur qu'il devait finir en 26 jours. Anna Snitkina savait bien qui était Dostoïevski, elle aimait ses livres et en admirait l'auteur. Pour elle, c'était un véritable honneur de travailler pour lui. Dostoïevski de son côté comprit vite les atouts de sa jeune sténographe (elle avait vingt ans de moins que lui, c'est-à-dire la même différence d'âge qu'entre ses parents), et quelques mois plus tard il la demanda en mariage. Ils se marièrent en avril $1867^{2}$.

Après le mariage, la jeune femme prit très vite en mains toute l'« entreprise Dostoïevski " (qui incluait l'œuvre et la famille). Dostoïevski la laissait faire. Il comprenait que c'était le mieux pour lui et pour tout son entourage. Pendant les quatorze ans de leur vie conjugale, Anna Grigorievna réussit à organiser la vie quotidienne de la famille.

Dans les matériaux autobiographiques publiés et les lettres on peut observer comment la jeune femme fière et indépendante s'est soumise aux exigences de son mari, comment elle acceptait son rôle en tant que maitresse de maison qui reste dans l'ombre de son mari célèbre. Très rarement elle l'accompagnait dans des salons et à des rendez-vous publics. Les seules exceptions eurent lieu durant les dernières années, quand Dostoïevski était devenu une célébrité nationale. Mais même lors de ces apparitions publiques, elle s'en tenait au rôle d'une infirmière, de l'épouse dévouée du grand écrivain. Elle n'était pas une mondaine, cette position ne l'intéressait pas; en outre, elle n'avait ni les moyens, ni l'envie d'acheter des vêtements élégants.

Il semble que pour elle l'argent soit resté secondaire. Anna Grigorievna avait gardé les mœurs et la façon de vivre de ses racines familiales, où la mère et la grand-mère conservaient les habitudes de la bourgeoisie moyenne, où les femmes travaillaient activement pour le bien-être de la famille. La fille de Dostoïevski (Lioubov) soulignait ce trait de caractère chez sa mère :

ШВЕДКИ РЕДКО ТЕРЯЮТ ПОЧВУ ПОД НОГАМИ. ВМЕСТО ПУСТЫХ МЕЧТАНИЙ МАТЬ СРАЗУ ЖЕ ВЗЯЛАСЬ ЗА ДЕЛО И НАЧАЛА С ТОГО, ЧТО СПАСЛА ОТЦА ИЗ КОГТЕЙ ЕГО ИЗДАТЕЛЕЙ. (

Dostoevskaâ, 1992 : 113) 
Habituée à travailler pour ses proches dès la maladie de son père et après sa mort, il était naturel pour elle de s'occuper du bien-être de sa propre famille. Comment concilier ce dévouement avec ses convictions de femme moderne?

Dans ses Mémoires Anna Grigorievna se présente comme une femme émancipée :

ОДНИМ ИЗ ПОВОДОВ НАШИХ ИДЕЙНЫХ РАЗНОГЛаСИЙ БЫЛ ТАК НАЗЫВАЕМЫЙ «ЖЕНСКИЙ ВОПРОС». БУДУЧИ ПО ВОЗРАСТУ СОВРЕМЕННИЦЕЙ ШЕСТИДЕСЯТЫХ ГОДОВ, Я ТВЕРДО СТОЯЛА ЗА ПРАВА И НЕЗАВИСИМОСТЬ ЖЕНЩИН И НЕГОДОВАЛА НА МУЖА ЗА ЕГО, ПО МОЕМУ МНЕНИЮ, НЕСПРАВЕДЛИВОЕ ОТНОШЕНИЕ К НИМ. ЯДАЖЕ ГОТОВА БЫЛА ПОДОБНОЕ ОТНОШЕНИЕ СЧИТАТЬ ЗА ЛИЧНУЮ ОБИДУ [...]. (Dostoevskaâ, 1971 : 163)

D'un côté elle se comportait comme une femme moderne, et de l'autre elle subissait sans protester la nature capricieuse de son mari.

On peut penser qu'elle sacrifiait son esprit émancipé pour le service d'un génie car, comme le disait Guippius, elle aimait davantage l'œuvre que l'homme. Elle supportait le créateur pour préserver et encourager la création. Dans sa façon de mener les affaires commerciales, on reconnaissait quand même le nouvel esprit bourgeois.

9 Il faut constater que les mœurs de la haute société avaient encore une grande influence sur la vie littéraire en Russie dans la deuxième moitié du XIX ${ }^{e}$ siècle.

Même si les salons littéraires aristocratiques s'étaient transformés en cercles de l'intelligentsia et si le marché des livres était en train de se former, les écrivains les plus célèbres comme Tolstoï et Tourguenïev étaient des nobles et menaient une vie conforme à la tradition. Tout en se révoltant contre les règles, ils restaient dans le cadre des conventions de leur classe. On le verra par exemple chez le couple Tolstoï - Tolstaïa.

21 Ainsi ce n'est pas un hasard si c'est Dostoïevskaïa, qui venait d'un milieu bourgeois, qui a été la première femme à s'occuper de la vente des œuvres de son mari. Pour ce faire, elle ouvrit un commerce à domicile qui se révéla de plus en plus profitable.

2 Elle dirigeait ses efforts sur trois domaines: créer des conditions pour une vie quotidienne normale dans la famille, en améliorer la situation économique et augmenter le prestige de l'écrivain. Elle faisait tout ce qui était en son possible pour en donner une image idéale, ce dont témoignent ses Mémoires [Vospominaniâ] qu'elle écrivit pendant les années 1911-1916.

Ces Mémoires montrent une vie familiale assez harmonieuse - plus harmonieuse qu'elle n'était en réalité - dans laquelle la première place est occupée par le grand auteur. Ellemême, son propre rôle, ses contributions au succès restent dans l'ombre.

Pendant les quatorze années de leur vie conjugale, elle a surmonté tous les problèmes familiaux : elle a su payer les dettes du frère de son mari, subvenir aux besoins du fils de la première femme, qui était très exigeant (et opposé au nouveau mariage de son beaupère), payer les dettes de jeu accumulées par son mari pendant les années de son séjour en Allemagne, mais aussi nourrir et éduquer ses propres enfants, ce qui n'était pas du tout simple.

Malgré tous ses efforts pour préserver dans ses Mémoires la réputation de son mari, on sent de temps en temps percer son désespoir. Voici un exemple :

МНЕ НУЖНО ЧТО-НИБУДЬ КУПИТЬ, Я ХОЖУ В РВАНОМ ПЛАТЬЕ, В ЧЕРНОМ ГАДКОМ ОДЕТА; НО Я ЕМУ НИЧЕГО НЕ ГОВОРЮ... Я ДУМАЮ, АВОСЬ ОН САМ ДОГАДАЕТСЯ, АВОСЬ САМ СКАЖЕТ, ЧТО ВОТ НАДО И ТЕБЕ КУПИТЬ ПЛАТЬЕВ ЛЕТНИХ, ВЕДЬ О СЕБЕ ОН ПОЗАБОТИЛСЯ И КУПИЛ В БЕРЛИНЕ, И В ДРЕЗДЕНЕ ЗАКАЗАЛ ПЛАТЬЕ, А У НЕГО ТОГДА НЕ ХВАТИЛО ЗАБОТЫ О ТОМ, ЧТО И МНЕ СЛЕДОВАЛО БЫ СДЕЛАТЬ, ЧТО Я ТАК СКВЕРНО ОДЕТА. (Ajhenval'd, 1925 : 150) 
Dostoïevskaïa ne se plaint pas de son destin, elle l'accepte comme un devoir, mais elle avoue dans son texte autobiographie que la période la plus heureuse de sa vie, malgré tous les problèmes pécuniaires, était les années passées avec son mari à Dresde, où elle sortait avec lui pour visiter les musées, les riches galeries et les parcs merveilleux. Là-bas elle n'était pas seulement la nourrice et la sténographe qui transmettait les notes prises pendant la journée, elle était la compagne de son mari, celle qui partageait sa vie intellectuelle et culturelle.

Il est remarquable qu'elle insiste comme résumé de ses Mémoires sur le fait qu'elle et son mari ont conservé durant toute leur vie commune des personnalités assez différentes :

ДЕЙСТВИТЕЛЬНО, МЫ С МУЖЕМ ПРЕДСТАВЛЯЛИ СОБОЙ ЛЮДЕЙ СОВСЕМ ДРУГОЙ

КОНСТРУКЦИИ, ДРУГОГО СКЛАДА, ДРУГИХ ВОЗЗРЕНИЙ [...] ВСЕГДА ОСТАВАЛИСЬ СОБОЙ. (

Dostoevskaâ, $1971: 35$ )

On comprend, en lisant ses Mémoires dans quelle mesure elle était tiraillée entre, d'un côté son désir d'une vie bourgeoise dans le cercle familial privé, et de l'autre son devoir de répondre aux exigences de son mari-écrivain, en tant que personnage public. Les scènes dans ses Mémoires qui décrivent la mort de son mari en témoignent. Elle se plaint de n'avoir eu aucune possibilité de rester seule auprès de son mari décédé. Il n'y eut aucun moment pour un adieu intime, les gens de la rue venaient sans cesse et transformaient la mort du génie en un événement public, sans prêter attention aucune à sa femme en deuil dans la chambre à côté.

Cela ressemble un peu à la situation à la gare d'Astapovo, où Sofia Andreïvna fut contrainte à une attente éprouvante et humiliante dans un wagon de train, car on voulait l'empêcher de voir son mari à l'article de la mort.

Toutefois, les deux scènes revêtent des significations bien différentes : tandis que la mort de Tolstoï est un événement public, pendant lequel les deux partenaires jouent leur rôle tragique, Dostoïevski, lui, meurt en privé, dans sa maison, qui se transforme au moment de la mort en espace public où l'on n'a plus besoin de la gardienne de la maison.

Dostoïevskaïa restait volontairement dans l'ombre, elle ne cherchait pas le public, mais sa vocation à organiser les affaires commerciales de son mari la fit entrer inévitablement dans la sphère publique, ce qui changea l'ordre établi de la vie littéraire en Russie.

Après la mort de Dostoïevski (en 1881) sa femme vécut encore presque quarante ans. Elle devint la veuve de Dostoïevski, celle que Guippius a rencontré en 1911: une femme autoritaire, mais qui ne cherchait pas la gloire personnelle.

Elle fut la première femme d'un écrivain non fonctionnaire à toucher une pension d'État. Elle fut suivie par Sofia Andreïevna, qui après la mort de Tolstoï touchait également une rente viagère.

\section{L'épouse de Tolstoi}

Sofia Andreïevna Behrs (1844-1919) était la deuxième fille des huit enfants de la famille Behrs. Le père, Andreï Behrs, était le descendant d'un militaire d'origine allemande, qui sur invitation d'Élisabeth $\mathrm{I}^{\mathrm{re}}$, la fille de Pierre $\mathrm{I}^{\mathrm{er}}$, était venu en Russie pour réorganiser le service militaire. Pendant l'incendie de Moscou (1812) la famille perdit tous ses biens, et les deux fils de la famille furent obligés d'apprendre une profession. Ils choisirent la médecine tous les deux. Le premier fils, le père de Sofia, travaillait comme médecin à la 
cour de l'empereur, et la famille habitait dans un petit logement sur le territoire du Kremlin à Moscou. La mère de Sofia Andreïevna était descendante d'une famille de l'ancienne noblesse. Même si la famille dans laquelle grandit Sofia n'était pas riche, elle gardait un style de vie aristocratique avec treize serviteurs. Comme les parents savaient que les trois filles seraient obligées de gagner leur vie, ils leurs donnèrent une solide formation. Cela se passait à un niveau plus élevé que dans la famille Snitkine. Les sœurs Behrs apprenaient des langues étrangères (français et allemand), elles pratiquaient les beaux-arts, la littérature, la danse et la musique et fréquentaient l'opéra et le théâtre. Une partie importante de l'éducation des sœurs était consacrée aux domaines pratiques : faire la cuisine, la couture, surveiller l'économie de la maison. À l'âge de seize ans Sofia réussit l'examen pour devenir institutrice (de russe et de français) à l'université de Moscou (avec une très bonne note). Sa dissertation était la meilleure de sa promotion. Dans son autobiographie de 1913, elle souligne l'atmosphère intellectuelle qui régnait dans les cercles d'étudiants, l'esprit des années soixante :

ЭТО БЫЛО НАЧАЛО ПРЕКРАСНЫХ ПО ДУХУ ШЕСТИДЕСЯТЫХ ГОДОВ! [...] МЫ, МОЛОДЫЕ, БЫЛИ

ПОЛНЫ ВОСТОРГА ПЕРЕД ВЕЛИКИМ СОБЫТИЕМ. (Ajhenval'd, 1925 : 9)

On s'intéressait aux courants nouveaux comme le matérialisme dans la philosophie, au nihilisme et à la libération des paysans.

En somme, Sofia Behrs grandissait dans des conditions typiques pour l'intelligentsia libérale. Avec cette formation - à la fois esthétique et pratique - elle était bien préparée pour remplir les devoirs multiples qui l'attendaient comme future femme d'un écrivain célèbre qui était en outre le propriétaire d'un grand domaine à la campagne.

Tolstoï était un ami de la famille Behrs, il avait de bonnes relations avec toutes les filles, beaucoup plus jeunes que lui. Il choisit finalement Sofia, qu'il trouvait séduisante et naïve, et dont il espérait faire une épouse conforme à son idéal patriarcal. Sofia avait seize ans de moins que lui. En septembre 1862 ils se marièrent à Moscou et partirent immédiatement pour commencer leur vie conjugale à la campagne.

Depuis le jour de son arrivée à Yasnaïa Poliana la vie de Sofia changea complètement. La jeune fille naïve et romantique, intéressée par les événements et centres culturels de la grande ville, les flirts, la musique et la danse, se transformait en « comtesse Tolstaïa », la maitresse de la maison, presque tout le temps enceinte, qui travaillait comme une esclave pour son mari et pour sa famille grandissante (seize naissances, et seulement neuf enfants restés en vie).

Son programme de travail comportait des tâches qui excédaient les forces d'une seule personne : elle devait remplir les devoirs d'une amante et d'une femme qui vivait dans des conditions patriarcales. De plus, c'était elle qui commençait l'éducation de ses enfants, tout en soutenant les efforts de son mari qui voulait fonder une école primaire pour les enfants du village. Elle participait également à la rédaction d'un manuel et travaillait comme institutrice à l'école de Yasnaïa Poliana.

Tolstoï était un grand propagandiste de la vie paysanne, mais un mauvais agriculteur. Sofia réussit à améliorer l'état misérable dans lequel se trouvait le grand domaine au moment de leur mariage. À partir de 1890, elle en était la seule propriétaire et portait donc la responsabilité de toute la gestion du domaine. En même temps, elle restait l'assistante de son mari et ménageait ses affaires littéraires. Le succès commercial de l'œuvre littéraire depuis Guerre et Paix est à mettre à son actif. Le livre se vendait avec profit et permettait à la famille une vie sans problèmes financiers. Sofia Andreïevna ne se 
contentait pas du rôle de copiste, mais donnait ses commentaires et ses conseils par rapport aux textes. En outre, elle était la seule personne capable de lire l'écriture de son mari, ainsi que ses innombrables corrections.

41 Tant qu'elle aimait son mari et l'adulait comme grand écrivain, tout allait bien. Elle regrettait de temps en temps de devoir vivre loin des plaisirs du monde, mais en général, elle était fière qu'un homme célèbre comme Tolstoï l'ait choisie comme assistante et comme épouse. Elle était très contente d'avoir le titre de comtesse. À cet égard, elle se distingue de Dostoïevskaïa, qui n'avait pas pour idéal la vie de la noblesse, urbaine ou provinciale, mais qui cherchait une vie familiale, loin du grand monde.

Tolstaïa, jeune fille, pratiquait elle-même la littérature, mais elle brûla son premier récit « Natacha » avant son mariage. Elle avait décidé de consacrer toutes ses forces à servir son mari, mais plus tard, quand leur relation fut détruite, elle se remit à travailler à son ouvrage littéraire. Comme Dostoïevskaïa elle tenait durant toutes ces années un journal, dans lequel on peut suivre sa vie de femme de Tolstoï : cette vie montre une rupture significative après la crise de 1881 . Tolstaïa se soumettait aux demandes de son mari tant qu'elle était en harmonie avec lui, quoiqu'elle ne partageât pas son image patriarcale de la femme. Quand Tolstoï abandonna ses idéaux littéraires pour se consacrer aux idées religieuses puritaines, elle se souvint de ses propres désirs et talents et se rebella contre son mari.

СЛУЖИЛА И Я, СОРОК ЛЕТ СКОРО ГЕНИЮ И ЗНАЮ КАК СОТНИ РАЗ ПОДНИМАЛАСЬ ВО МНЕ УМСТВЕННАЯ ЖИЗНЬ, ВСЯКИЕ ЖЕЛАНИЯ, ЭНЕРГИЯ, СТРЕМЛЕНИЕ К РАЗВИТИЮ, ЛЮБОВЬ К ИСКУССТВАМ, К МУЗЫКЕ... И ВСЕ ЭТИ ПОРЫВЫ Я ПОДАВЛЯЛА И ГЛУШИЛА И ОПЯТЬ, И ОПЯТЬ, И ТЕПЕРЬ, И ДО КОНЦА ЖИЗНИ БУДУ ТАК ИЛИ ИНАЧЕ СЛУЖИТЬ СВОЕМУГЕНИЮ. (Note du 13 mars 1902, dans Tolstaâ [1978, t. 2 : 61])

Néanmoins, elle ne cessa jamais de défendre l'œuvre littéraire de son mari, et de faire tout son possible pour le publier.

Bien sûr, elle était en concurrence avec le disciple de Tolstoï, Chertkov, qui voulait populariser les idées de son idole dans une édition populaire, tandis que la comtesse s'efforçait de garder la propriété de l'œuvre littéraire de son mari pour la famille et pour ses enfants. De plus, elle faisait tout pour défendre la réputation de Tolstoï en tant qu'artiste contre Chertkov et ses semblables, qui considéraient l'écrivain comme un idéologue.

On peut donc dire que Sofia Andreïevna se comportait d'une façon semblable à Dostoïevskaïa en ce qui concerne l'œuvre littéraire. Elle aussi passait ses nuits à mettre au propre les manuscrits de son mari (au minimum sept copies de Guerre et Paix, plus de huit d'Anna Karénine) et elle s'occupait de l'édition et du commerce de ses livres. Pour réaliser ce projet elle empruntait de l'argent à sa mère (10000 roubles) et à un voisin noble (15000 roubles). Elle consultait Dostoïevskaïa qui lui servait de modèle en ce qui concerne l'organisation d'un commerce efficace (Nikitina, 2010:155-157). En 1881, Sofia Andreïevna acheta une maison à Moscou, où elle ouvrit une petite librairie (Wilson, 1988 : 352). Pour conserver les manuscrits et les matériaux de travail de Tolstoï pour la postérité, elle organisa leur transfert au Musée historique (en 1904) à Moscou :

НАДО СПАСТИ ВСЕ, ЧТО МОЖНО, ОТ БЕСТОЛКОВОГО РАСХИЩЕНИЯ ВЕЩЕЙ ДЕТЬМИ И ВНУКАМИ. (Note du 18 janvier 1904, dans Tolstaâ [1978, t. 2 : 100])

Tolstaïa travaillait sans cesse : elle menait une lutte acharnée pour sauver le domaine pour ses enfants, pour la famille, contre les idéalistes, les disciples de Tolstoï, et même contre les convictions de son mari, qui condamnait la propriété privée et qui voulait s'en 
libérer. Sofia Andreïevna créait les conditions nécessaires de travail pour l'écrivain Tolstoï, mais elle se rebella contre Tolstoï en tant qu'idéologue :

ВСЯ ТЯЖЕСТЬ ДОБЫВАНИЯ СРЕДСТВ, ХОЗЯЙСТВА, ПЕЧАТАНЬЕ КНИГ - ВСЕ ЛЕЖИТ НА МНЕ, ЧТОБ ВСЮ ЖИЗНЬ ДАВАТЬ ЛЬВУ НИК. СПОКОЙСТВИЕ, УДОБСТВО И ДОСУГ ДЛЯ ЕГО РАБОТ. ( Note du 31 juillet 1910, dans Tolstaâ [1978, t. 2 : 163])

Avec les années, elle devint de plus en plus insatisfaite de son destin. En particulier, le fait que son mari l'ait obligée deux fois à mener à terme une grossesse, quoique sa santé fût menacée, la rendait amère. Tolstoï voulait mettre à réalisation, sur l'exemple de sa femme, son idéal féminin de proximité avec la nature animale (comme femelle). Pour la démonstration littéraire de cet idéal, il avait créé le personnage de Natacha Rostov.

En ce qui concerne sa place dans la vie publique, Sofia Andreïevna était, certes, plus visible que Dostoïevskaïa. Elle le devait à sa propre nature ardente, mais aussi à la réputation grandissante de son mari, comme prophète et saint hérétique. Sa femme, même si elle ne partageait pas ses idées, ou, peut être justement parce qu'elle s'y opposait, occupait une position importante dans la société russe. Elle en était consciente et elle utilisait sa popularité pour soutenir celles des activités de son mari avec lesquelles elle était d'accord. Voici deux exemples :

1. Pendant la grande famine en Russie centrale et méridionale en 1891, Tolstoi s'engagea avec toute sa famille pour une large action de charité dans tout le pays. Tolstaïa publia un appel aux dons pour les victimes dans le journal Russkie vedomosti. Cet appel fut publié en Russie, en France, en Angleterre et en Amérique. En peu de temps, Tolstaïa parvenait à récolter treize mille roubles. Dans son appel, Sofia Andreïevna se réfère à son statut d'épouse de Tolstoï et de membre d'une entreprise familiale célèbre :

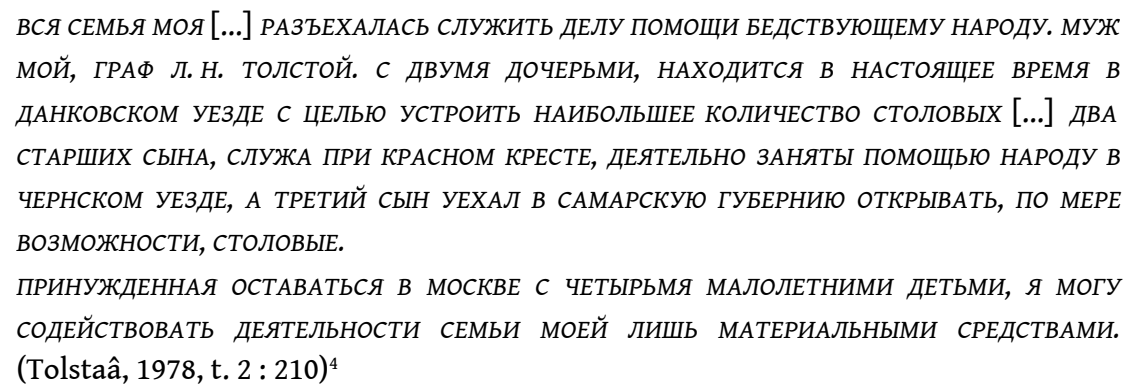

2. Elle luttait à tout prix pour l'édition des œuvres littéraires de son mari en se servant de son autorité personnelle. Quand la censure stoppa l'édition du tome XIII des Euvres littéraires, à cause du récit «La Sonate à Kreutzer » (1889), qui avait provoqué un scandale et offensé également les sentiments de Sofia Andreïevna, elle se rendit pourtant à SaintPétersbourg pour faire avancer les choses. Elle rendit visite au comité de censure et persuada le fonctionnaire de service d'annuler l'interdiction du récit, elle obtint même une audience privée auprès de l'empereur Alexandre III (le 13 avril 1890). Le tsar se montra impressionné par la comtesse Tolstaïa et donna la permission de publier le récit dans le tome XIII de la collection complète. Tolstaïa avait de quoi être fière de son succès.

\section{Conclusion}

Cette brève comparaison entre deux épouses d'écrivains connus nous montre d'abord qu'il faut prendre en considération les différences concernant leur milieu social et leur tempérament: les Dostoïevski vivaient dans une ambiance bourgeoise, tandis que les 
Tolstoï appartenaient à la noblesse, ce qui ne pouvait manquer d'avoir une influence sur leurs mœurs et leur comportement. idéaux de l'amour et de la famille, et de s'adapter a la réalité. La vie a côté d'un génie n'était pas toujours exaltante, mais plus souvent exigeante et pleine de difficultés. En outre, les jeunes femmes modernes étaient confrontées à des conceptions traditionnelles de la relation entre les sexes. Ni Dostoïevski, ni Tolstoï n'acceptaient les «femmes nouvelles», ils ironisaient sur les «femmes nihilistes" et défendaient un modèle familial patriarcal. Les jeunes femmes essayèrent de protester, mais leur protestation restait faible et fut vite avalée par les exigences féroces de la vie quotidienne. elles contribuaient activement à modeler son image publique pour la postérité. Elles devinrent elles-mêmes des écrivains, publiant chacune leurs carnets, leurs lettres et leurs mémoires. consciemment) que leur vie se déroulerait en public.

Leur image publique, elles la doivent à la célébrité de leur mari, mais il ne faut pas oublier que le mariage était encore, en Russie au xIx ${ }^{\mathrm{e}}$ siècle, presque la seule possibilité pour une femme de gagner une place visible dans la société.

56

N'oublions pas non plus que c'est aussi grâce à elles que Tolstoï et Dostoïevski sont devenus ce qu'ils sont comme auteurs littéraires. Loin d'être une exception, on retrouve encore aujourd'hui le modèle où l'épouse fait partie de «l'institution littéraire publique » que représente un écrivain ou un poète célèbre. 


\section{BIBLIOGRAPHIE}

AJHENVAL'D Ûrij I. (1925), Dve ženy. Tolstaâ i Dostoevskâa. Materialy i kommentarii [Deux épouses.

Tolstaïa et Dostoevskaïa], Berlin : Izdatel'stvo Pisatelej.

AsQUITH Cynthia (1960), Married to Tolstoy [Mariée avec Tolstoï], Londres: Hutchinson.

DostoevsKaÂ Anna Grigor'evna (1971), Vospominaniâ [Mémoires], Moscou : Hudožestvennaâ literatura (Seriâ literaturnyh memuarov).

DostoevsKaÂ Lidiâ Fedorovna (1992), Dostoevskij v izobraženii svoej dočeri [Dostoïevski dans la représentation de sa fille], Saint-Pétersbourg : Andreev i synov'â.

DostojeWsKi Fjodor \& DostojeWsKAJA Anna (1982), Briefwechsel 1866-1880 [Correspondance 1866-1880], Berlin : Rütten \& Loening.

GIPPIUS Zinaida (1991), «O ženah » [À propos des épouses], Russkij éros ili filosofiâ ljubvi v Rossii [L'éros russe ou la philosophie de l'amour en Russie], Moscou : Progress, 215-220.

Keller Ursula \& ShaRANDAK Natalja (2010), Sofja Andrejewna Tolstaja. Ein Leben an der Seite Tolstojs [Sofia Andreïevna Tolstaïa. Une vie à côté de Tolstoï], Berlin : Insel Verlag.

NiKitinA Nina (2010), Sof â Tolstaâ, Moscou : Molodaâ gvardiâ (Žizn' zamečatel'nyh lûdej).

TolsTAÂ Sof'â Andreevna (1978), Dnevniki w dvuh tomah [Journaux en deux tomes], Moscou :

Hudožestvennaâ literatura.

Tolstor Lew \& TolStAjA Sofja (2010), Eine Ehe in Briefen [Mariage en lettres], Berlin : Insel Verlag.

WiLSON A. N. (1988), Tolstoy, Londres : Penguin Group.

\section{NOTES}

1. « Я ПРИНАДЛЕЖАЛА К ЛИБЕРАЛЬНОМУ ПОКОЛЕНИЮ 60-Х ГОДОВ. » (Dostoevskaâ, 1971 : 49)

2. C'était le deuxième mariage de Dostoïevski, dont la première épouse était morte en 1864.

3. La fille de Dostoïevski exagère évidemment en disant que l'origine suédoise eut une influence prépondérante sur le caractère de sa famille.

4. Cf. aussi Nikitina (2010: 189-190).

\section{RÉSUMÉS}

La femme d'un poète ou d'un écrivain occupe une position intermédiaire et ambiguë : elle a une vie publique dans sa fonction d'épouse de poète - et en tant que telle elle est considérée et jugée par les historiens de la littérature. En même temps, elle occupe une place dans la vie quotidienne, 
où elle doit organiser la vie de famille, le confort et les conditions agréables pour son mari. Concernant cette fonction elle partage le destin de toutes les femmes de son milieu et de son époque. Le jugement porté par la critique ou par la presse sur le comportement des épouses des poètes Dostoïevski et de Tolstoï est assez controversé.

Cette intervention a pour objet de regarder les conditions familiales et sociales dans lesquelles les deux femmes exprimaient leur personnalité, comment elles trouvaient leur place dans la vie quotidienne. Enfin il sera question du rôle qu'elles jouaient pour la genèse et la diffusion de l'œuvre de leur célèbre mari.

ЖЕНА ПОЭТА ИЛИ ПИСАТЕЛЯ ЗАНИМАЕТ ПРОМЕЖУТОЧНОЕ И НЕОДНОЗНАЧНОЕ ПОЛОЖЕНИЕ В ОБЩЕСТВЕ. ЕЕ ЖИЗНЬ ДЕЛИТСЯ НА ДВЕ СФЕРЫ: ОФИЦИАЛЬНУЮ КАК ЖЕНА ПОЭТА И ЛИЧНУЮ КАК ХОЗЯЙКА СЕМЕЙНОЙ ЖИЗНИ. В ЭТОЙ ПОСЛЕДНЕЙ ФУНКЦИИ ОНА РАЗДЕЛЯЕТ СУДЬБУ СВОИХ СОВРЕМЕННИЦ, СХОДНЫХ ПО СОСЛОВИЮ И ОБЩЕСТВЕННОМУ ПОЛОЖЕНИЮ В РОССИИ ВТОРОЙ ПОЛОВИНЫ 19 ОrО ВЕКА. ОЦЕНКА ДОСТОЕВСКОЙ И ТОЛСТОЙ СО СТОРОНЫ КРИТИКОВ ДОВОЛЬНО СПОРНА, ЧАЩЕ ВСЕГО ОБЕ ЖЕНЩИНЫ РАССМАТРИВАЮТСЯ ЛИШЬ В РОЛИ ЖЕНЫ ПОЭТА, А НЕ КАК САМОСТОЯТЕЛЬНЫЕ ЛИЧНОСТИ.

СТАТЬЯ ПРЕДПРИНИМАЕТ ПОПЫТКУ СОЕДИНИТЬ ЭТИ ДВЕ СФЕРЫ. В РАМКАХ СТАТЬИ РАССМАТРИВАЮТСЯ КОНКРЕТНЫЕ УСЛОВИЯ, В КОТОРЫХ ДОСТОЕВСКАЯ И ТОЛСТАЯ ФОРМИРОВАЛИ СВОЮ ЛИЧНОСТЬ; ПРОСЛЕЖИВАЮТСЯ ПРОЦЕССЫ ПОИСКА СВОЕГО МЕСТА В ТАКОЙ СПЕЦИФИЧЕСКОЙ ОРГАНИЗАЦИИ, КОТОРОЙ ЯВЛЯЕТСЯ СЕМЬЯ ПИСАТЕЛЯ, А ТАКЖЕ УТОЧНЯЕТСЯ, КАКУЮ РОЛЬ ЖЁНЫ ПИСАТЕЛЕЙ ИГРАЛИ В ПРОЦЕССЕ ТВОРЧЕСТВА И РАСПРОСТРАНЕНИЯ ТРУДОВ СВОИХ ЗНАМЕНИТЫХ МУЖЕЙ.

\section{INDEX}

Mots-clés : histoire de la littérature russe, Tolstoï et Dostoïevski - informations biographiques, histoire des femmes russes au XIXe siècle

motsclesru ИСТОРИЯ РУССКОЙ ЛИТЕРАТУРЫ, ТОЛСТОЙ И ДОСТОЕВСКИЙ - БИОГРАФИЧЕСКИЕ ДАННЫЕ, ИСТОРИЯ ЖЕНЩИН В РОССИИ 19 ОГО ВЕКА

\section{AUTEUR}

\section{CHRISTA EBERT}

Université européenne de Francfort-sur-l'Oder (Allemagne) 